\title{
Comprehensive Laboratory Data Analysis to Predict the Clinical Severity of Coronavirus Disease 2019 in 1,952 Patients in Daegu, Korea
}

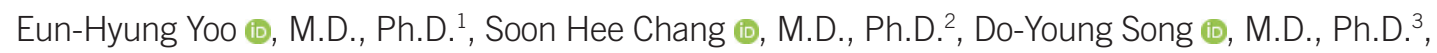

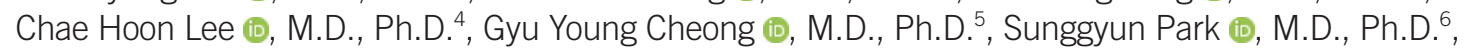

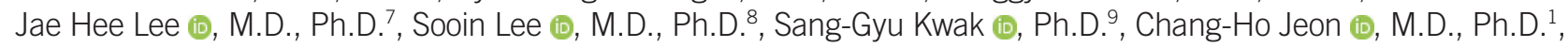 \\ and Kyung Eun Song $\mathbb{i}$, M.D., Ph.D. ${ }^{2}$

\begin{abstract}
${ }^{1}$ Department of Laboratory Medicine, Daegu Catholic University School of Medicine, Daegu, Korea; ${ }^{2}$ Department of Clinical Pathology, Kyungpook National University School of Medicine, Daegu, Korea; ${ }^{3}$ Department of Laboratory Medicine, Daegu Fatima Hospital, Daegu, Korea; ${ }^{4}$ Department of Laboratory Medicine, Yeungnam University College of Medicine, Daegu, Korea; ${ }^{5}$ Department of Laboratory Medicine, Daegu Medical Center, Daegu, Korea; ${ }^{6}$ Department of Laboratory Medicine, Keimyung University School of Medicine, Daegu, Korea; ${ }^{7}$ Department of Laboratory Medicine, Keimyung University Daegu-Dongsan Hospital, Daegu, Korea; ${ }^{8}$ Department of Laboratory Medicine, Daegu Veterans Hospital, Daegu, Korea; ${ }^{9}$ Department of Medical Statistics, Daegu Catholic University School of Medicine, Daegu Korea
\end{abstract}

Background: Laboratory parameter abnormalities are commonly observed in COVID-19 patients; however, their clinical significance remains controversial. We assessed the prevalence, characteristics, and clinical impact of laboratory parameters in COVID-19 patients hospitalized in Daegu, Korea.

Methods: We investigated the clinical and laboratory parameters of 1,952 COVID-19 patients on admission in nine hospitals in Daegu, Korea. The average patient age was 58.1 years, and 700 (35.9\%) patients were men. The patients were classified into mild ( $N=1,612)$, moderate $(N=294)$, and severe $(N=46)$ disease groups based on clinical severity scores. We used chi-square test, multiple comparison analysis, and multinomial logistic regression to evaluate the correlation between laboratory parameters and disease severity.

Results: Laboratory parameters on admission in the three disease groups were significantly different in terms of hematologic ( $\mathrm{Hb}$, Hct, white blood cell count, lymphocyte\%, and platelet count), coagulation (prothrombin time and activated partial thromboplastin time), biochemical (albumin, aspartate aminotransferase, alanine aminotransferase, lactate, blood urea nitrogen, creatinine, and electrolytes), inflammatory (C-reactive protein and procalcitonin), cardiac (creatinine kinase MB isoenzyme and troponin I), and molecular virologic (Ct value of SARS-CoV-2 RdRP gene) parameters. Relative lymphopenia, prothrombin time prolongation, and hypoalbuminemia were significant indicators of COVID-19 severity. Patients with both hypoalbuminemia and lymphopenia had a higher risk of severe COVID-19.

Conclusions: Laboratory parameter abnormalities on admission are common, are significantly associated with clinical severity, and can serve as independent predictors of COVID-19 severity. Monitoring the laboratory parameters, including albumin and lymphocyte count, is crucial for timely treatment of COVID-19.

Key Words: Hypoalbuminemia, Lymphopenia, Prothrombin time, Coronavirus disease 2019, Severity, Laboratory parameters
Received: November 2, 2020

Revision received: February 27, 2021

Accepted: July 26, 2021

\section{Corresponding author:}

Kyung Eun Song, M.D., Ph.D.

Department of Clinical Pathology,

Kyungpook National University School of

Medicine, 680 Gukchebosang-ro, Jung-gu,

Daegu 41944, Korea

Tel: +82-53-200-3306

Fax: +82-53-426-3367

E-mail: kesong@knu.ac.kr (c) Korean Society for Laboratory Medicine This is an Open Access article distributed under the terms of the Creative Commons Attribution Non-Commercial License (https://creativecommons.org/licenses/by-nc/4.0) which permits unrestricted non-commercial use, distribution, and reproduction in any medium, provided the original work is properly cited. 


\section{INTRODUCTION}

Daegu, South Korea, was host to the first coronavirus disease 2019 (COVID-19) outbreak outside of China. Since February 18,2020 , the number of infected patients increased exponentially, and by August 5, 2020, more than 6,943 confirmed cases (48.03\% of the total cases in South Korea) and 191 deaths were reported in Daegu [1, 2].

The initial clinical characteristics of COVID-19 are non-specific, and the clinical spectrum varies widely from asymptomatic, mild respiratory symptoms, and pneumonia to acute respiratory distress syndrome [3-5]. An exponential increase in the number of COVID-19 patients led to a shortage of hospital beds, healthcare supplies, and medical personnel. By early March, more than 2,000 patients were waiting for hospital beds, and three patients died at home in Daegu. Therefore, while the number of confirmed cases increases, rapid and strategic patient allocation according to clinical severity and proper treatment are critical to lower the mortality rate.

The Korean government has classified COVID-19 cases by severity to prioritize the treatment of more severe cases at hospitals, while asymptomatic patients and those with mild symptoms receive medical treatment and monitoring at community treatment centers. Depending on their condition, patients with more severe disease are admitted to infectious disease hospitals or nationally designated treatment facilities for immediate treatment [6]. Severity-based classification involves several indicators, such as consciousness level, body temperature, presence of pneumonia, age, and comorbidities in confirmed cases [7]. The clinical and laboratory parameters of COVID-19 have been analyzed to identify risk factors that can predict severity. Laboratory parameters may be more objective than clinical parameters for assessing patient condition. Several studies have attempted to identify laboratory parameters correlated with disease severity [8-10]. Abnormal results of laboratory parameters, such as Creactive protein $(C R P)$, lactate dehydrogenase $(L D)$, neutrophil: lymphocyte ratio, fibrinogen, D-dimer, interleukin (IL)-6, platelet count, and lymphocyte count, are reportedly related to COVID-19 severity [8]. However, most of these results are from other countries or races and are based on meta-analysis. Thus, there is a need to identify predictive laboratory parameters in Korean COVID-19 patients.

To manage the next wave of the pandemic, complimentary strategies should be established for efficient epidemic management and patient care. We comprehensively analyzed laboratory parameters of COVID-19 patients in Daegu and determined their correlation with clinical disease severity to provide a basis for the development of improved treatment strategies.

\section{MATERIALS AND METHODS}

\section{Patients}

All hospitals providing COVID-19 treatment in Daegu were requested to submit clinical and laboratory data to the Korea Disease Control and Prevention Agency database, Internet-based Clinical Research and Trial (iCReaT). In total, 1,952 patients hospitalized at nine general hospitals in Daegu between February 17, 2020 and April 12, 2020 were enrolled retrospectively. Most patients were Korean (1,932; 99.0\%), fourteen were KoreanChinese, and six were of other nationalities. All patients were confirmed of having COVID-19 using the real-time reverse transcription (RT) PCR testing for severe acute respiratory syndrome coronavirus 2 (SARS-CoV-2) using upper and/or lower respiratory tract (URT/LRT) specimens.

Clinical severity was assessed on admission based on an eightcategory WHO ordinal scale modified by the Korea National Committee for Clinical Management of COVID-19: (1) no limitation in performing daily activities; (2) limitation in performing daily activities, but no need for supplemental oxygen therapy; (3) need for supplemental oxygen therapy via a nasal cannula; (4) need for supplemental oxygen therapy via facial mask; (5) need for highflow supplemental oxygen therapy or noninvasive mechanical ventilation; (6) need for invasive mechanical ventilation; (7) multiorgan failure or the need for extracorporeal membrane oxygenation (ECMO) therapy; and (8) death [11]. On admission, the clinical severity scores for the patients were 1-2 (mild), 3-4 (moderate), and 5-8 (severe) in 1,612 (82.6\%), 294 (15.1\%), and $46(2.4 \%)$ patients, respectively. The 294 patients in the moderate group were treated with supplemental oxygen therapy via a nasal cannula ( $\mathrm{N}=223)$ or facial mask $(\mathrm{N}=71)$. Of the 46 patients in the severe group, 37 were treated with either highflow oxygen/noninvasive mechanical ventilation $(N=21)$, mechanical ventilation $(\mathrm{N}=15)$, or $\mathrm{ECMO}(\mathrm{N}=1)$, while nine patients died on the day of admission. This study was approved by the Daegu Joint Institutional Review Board (2020-07-001) in agreement with the World Medical Association Declaration of Helsinki.

\section{Clinical and laboratory parameters}

We collected demographic and clinical information on age, sex, initial symptoms, comorbidities, and final outcomes (discharge, hospitalized, or death). On admission, we analyzed the following laboratory parameters: complete blood counts (CBC) [hemoglo- 
bin ( $\mathrm{Hb})$, hematocrit (Hct), lymphocyte (LYM)\%, white blood cell (WBC) count, platelet (PLT) count], coagulation profile [activated partial thromboplastin time (aPTT) and prothrombin time (PT) international normalized ratio (INR)], biochemical parameters [albumin, total bilirubin (T-BIL), alanine aminotransferase (ALT), aspartate aminotransferase (AST), blood urea nitrogen (BUN), creatinine ( $\mathrm{Cr}$ ), CRP, glucose (Glu), sodium (Na), chloride $(\mathrm{Cl})$, potassium $(\mathrm{K})$, lactate $(\mathrm{Lac}), \mathrm{LD}$, procalcitonin $(\mathrm{PCT})$ ], cardiac markers [creatinine kinase MB isoenzyme (CK-MB), troponin I (Tnl), troponin $T(T n T)$ ], and molecular marker for SARS-CoV-2 [Ct values of RNA-dependent RNA polymerase (RdRP) gene of SARS-CoV-2 RT-PCR]. We compared the clinical characteristics and laboratory parameters according to the clinical severity determined on admission.

\section{Statistical analysis}

Continuous variables are expressed as mean \pm standard deviation, while categorical variables are presented as frequencies and percentages. Groups of variables were compared using Chi-square test or one-way ANOVA. Multiple comparison analysis was performed using Scheffé's method [12]. Multinomial logistic regression analysis was used to evaluate the correlations between laboratory parameters and clinical severity on admission in COVID-19 patients. Two types of multinomial logistic regression analysis were used: one using the independent variables, i.e., the laboratory parameters, as quantitative variables and the other using the independent variables as qualitative variables, categorizing them as normal or abnormal based on their respective reference range.

Among the 26 laboratory parameters, albumin and LYM\% showed abnormality most frequently among the groups according to clinical severity, with approximately $80 \%$ of patients in the severe group showing abnormal results. Therefore, we also analyzed the correlations of hypoalbuminemia (albumin $<3.5 \mathrm{~g} / \mathrm{dL}$ ), lymphopenia (LYM\% <21\%), and combined hypoalbuminemia and lymphopenia with clinical severity. Odds ratios (ORs) with

Table 1. Baseline clinical characteristics of 1,952 COVID-19 patients in Daegu, Korea

\begin{tabular}{|c|c|c|c|c|c|c|}
\hline \multirow{2}{*}{ Variable } & & \multirow{2}{*}{$\begin{array}{l}\text { All patients } \\
(\mathrm{N}=1,952)\end{array}$} & \multicolumn{3}{|c|}{ Clinical severity on admission } & \multirow{2}{*}{$P$} \\
\hline & & & Mild $(\mathrm{N}=1,612)$ & Moderate $(\mathrm{N}=294)$ & Severe $(N=46)$ & \\
\hline Age (yr) & & $58.1 \pm 19.9$ & $55.3 \pm 20.0$ & $71.7 \pm 12.6$ & $70.1 \pm 14.5$ & $<0.001$ \\
\hline Male, N (\%) & & $700(35.9)$ & $536(33.3)$ & $137(46.6)$ & $27(58.7)$ & $<0.001$ \\
\hline \multirow[t]{9}{*}{ Symptoms, N (\%) } & Fever & $422(21.6)$ & $294(18.2)$ & $108(36.8)$ & $20(43.5)$ & $<0.001$ \\
\hline & Cough & $874(44.8)$ & $714(44.3)$ & $145(49.3)$ & $15(32.6)$ & $<0.001$ \\
\hline & Sputum & $677(34.7)$ & $555(34.4)$ & $111(37.8)$ & $11(23.9)$ & $<0.001$ \\
\hline & Sore throat & $242(12.4)$ & $215(13.3)$ & $24(8.2)$ & $3(6.5)$ & $<0.001$ \\
\hline & Rhinorrhea & $193(9.9)$ & $167(10.4)$ & $25(8.5)$ & $1(2.2)$ & $<0.001$ \\
\hline & Myalgia & $392(20.1)$ & $331(20.5)$ & $53(18.0)$ & $8(17.4)$ & $<0.001$ \\
\hline & Dyspnea & $402(20.6)$ & $213(13.2)$ & $158(53.7)$ & $31(67.4)$ & $<0.001$ \\
\hline & Headache & $389(19.9)$ & $348(21.6)$ & $39(13.3)$ & $2(4.4)$ & $<0.001$ \\
\hline & Diarrhea & $257(13.2)$ & $213(13.2)$ & $41(14.0)$ & $3(6.5)$ & $<0.001$ \\
\hline \multirow[t]{7}{*}{ Comorbidities, N (\%) } & Diabetes & $368(18.9)$ & 257 (15.9) & $97(33.0)$ & $14(30.4)$ & $<0.001$ \\
\hline & Hypertension & $619(31.7)$ & $443(27.5)$ & $151(51.4)$ & $25(54.4)$ & $<0.001$ \\
\hline & Asthma & $67(3.4)$ & $51(3.2)$ & $12(4.1)$ & $4(8.7)$ & $<0.001$ \\
\hline & COPD & $27(1.4)$ & $18(1.1)$ & $9(3.1)$ & $0(0)$ & $<0.001$ \\
\hline & CKD & $38(1.9)$ & $21(1.3)$ & $13(4.4)$ & $4(8.7)$ & $<0.001$ \\
\hline & CLD & $33(1.7)$ & $24(1.5)$ & $8(2.7)$ & $1(2.2)$ & $<0.001$ \\
\hline & Malignancy & $86(4.4)$ & 64 (3.97) & $16(5.44)$ & $6(13.0)$ & $<0.001$ \\
\hline \multirow[t]{3}{*}{ Final outcomes, N (\%) } & Discharged & $1,490(76.3)$ & 1,337 (82.9) & $146(49.7)$ & $7(15.2)$ & $<0.001$ \\
\hline & Hospitalized & $298(15.3)$ & $223(13.8)$ & $65(22.1)$ & $10(21.7)$ & \\
\hline & Death & $164(8.4)$ & $52(3.2)$ & $83(28.2)$ & $29(63.0)$ & \\
\hline
\end{tabular}

Data are presented as the mean \pm SD or $\mathrm{N}(\%)$.

Abbreviations: CKD, chronic kidney disease; CLD, chronic liver disease; COPD, chronic obstructive pulmonary disease. 
95\% confidence intervals ( $\mathrm{Cls}$ ) were calculated to examine the significance of correlations. The optimal cut-off values for albumin and LYM\% in predicting severity were evaluated using receiver operating characteristic (ROC) curve analysis. Data were analyzed using the SPSS Statistics 19.0 software (IBM corporation, Armonk, NY, USA). $P<0.05$ was considered statistically significant.

\section{RESULTS}

\section{Baseline clinical characteristics}

The baseline characteristics of the COVID-19 patients hospitalized in Daegu are summarized in Table 1. The mean age of the study population was $58.1 \pm 19.9$ years, and 700 (35.9\%) patients were men. The mean patient age in the mild group was
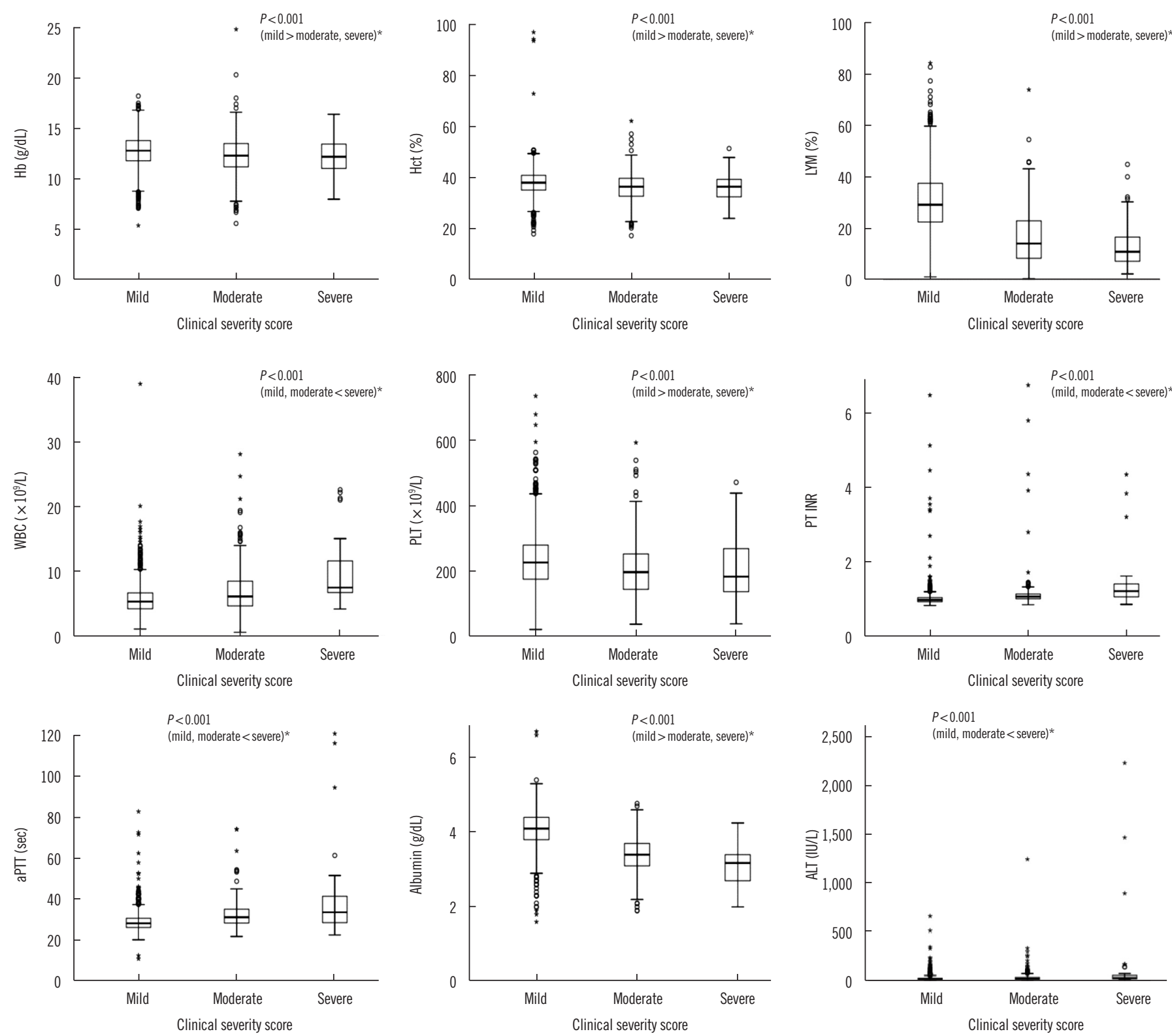

Fig. 1. Comparison of laboratory parameters based on clinical severity. * Multiple comparison result using Scheffé's method.

Abbreviations: ALT, alanine aminotransferase; aPTT, activated partial thromboplastin time; AST, aspartate aminotransferase; BUN, blood urea nitrogen; CK$\mathrm{MB}$, creatinine kinase MB isoenzyme; Cl, chloride; Cr, creatinine; CRP, C-reactive protein; Ct, cyclic threshold; Glu, glucose; K, potassium; Lac, lactate; LD, lactate dehydrogenase; LRT, lower respiratory tract; LYM, lymphocyte; Na, sodium; PCT, procalcitonin; PLT, platelet; PT INR, prothrombin time international normalized ratio; RdRP, RNA-dependent RNA polymerase gene; T-BIL, total bilirubin; Tnl, troponin I; TnT, troponin T; URT, upper respiratory tract; WBC, white blood cell.

(Continued to the next page) 

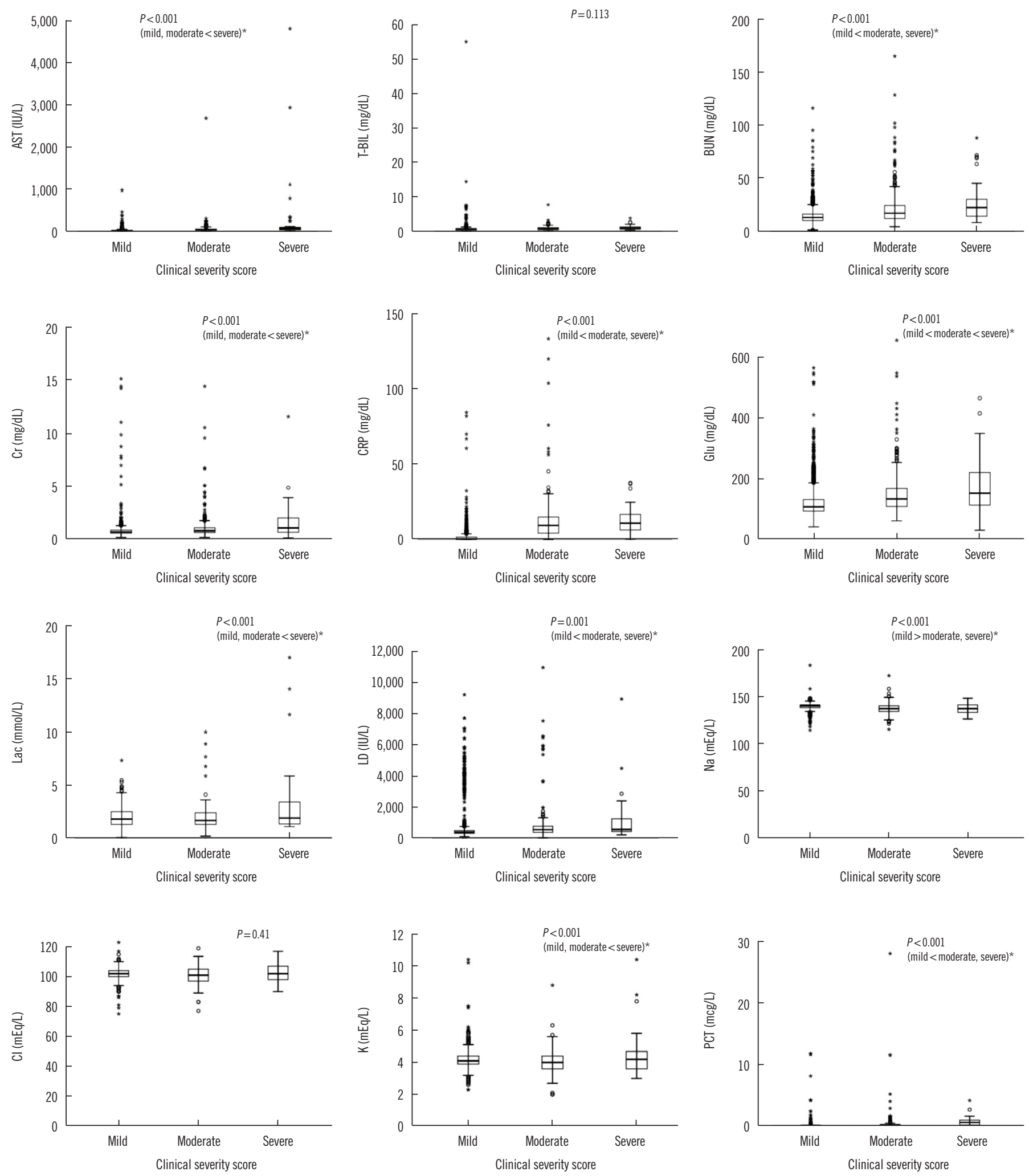

Fig. 1. Continued.

(Continued to the next page) 

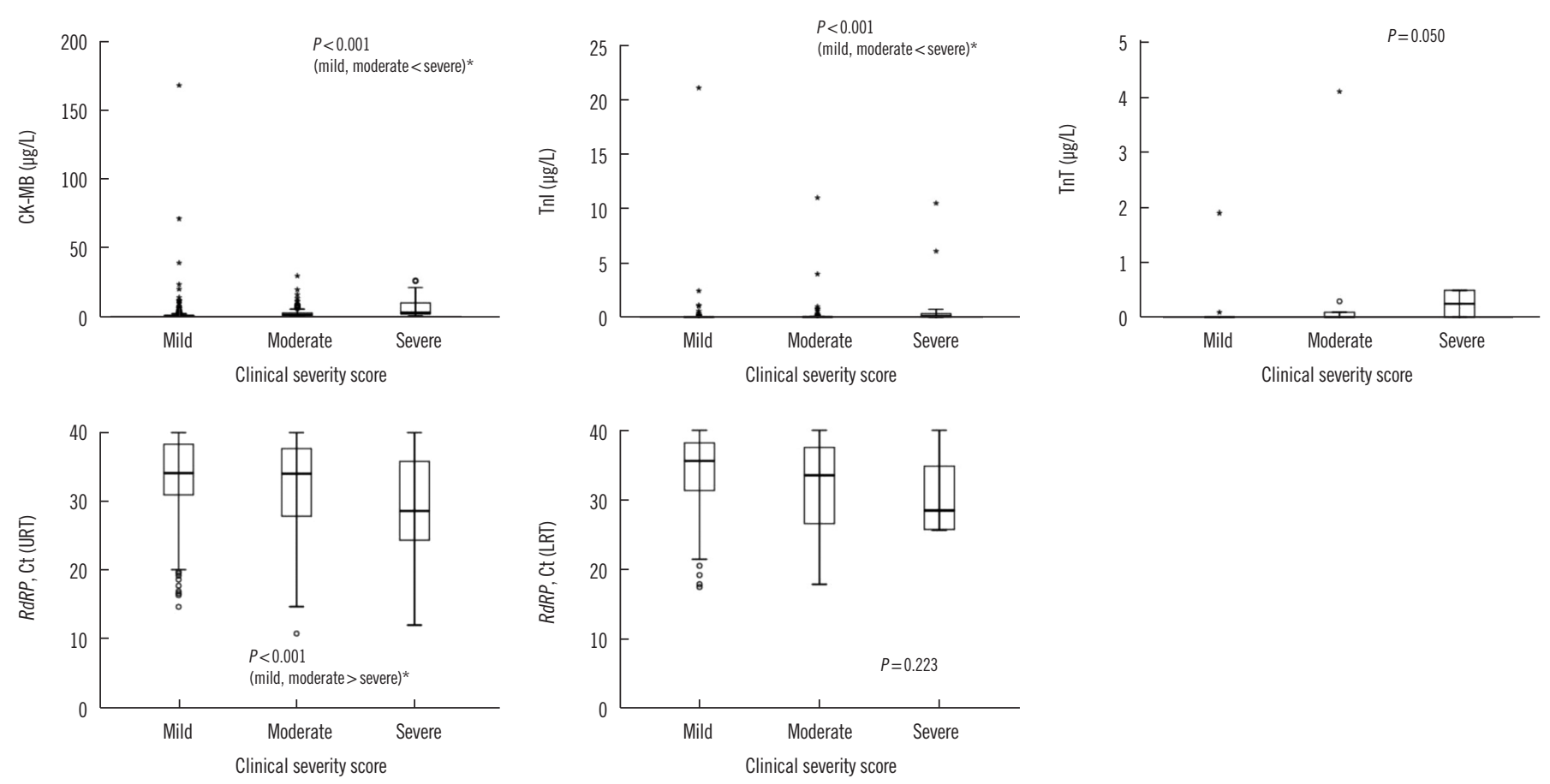

Fig. 1. Continued.

lower than that in the other groups. The most common symptom on admission was cough (874 patients, $44.8 \%$ ), followed by sputum (677 patients, $34.7 \%$ ), fever (422 patients, $21.6 \%$ ), and dyspnea (402 patients, 20.6\%). Fever and dyspnea were more common in the severe and moderate groups than in the mild group $(P<0.001)$. The most common comorbidity was hypertension (619 patients, $31.7 \%$ ), followed by diabetes (368 patients, $18.9 \%$ ) and malignancy (86 patients, $4.4 \%$ ). At the time of epidemiological data collection, $1,490(76.3 \%)$ of the 1,952 patients had been discharged, and 164 (8.4\%) patients had died. The proportions of outcomes significantly differed among the three groups $(P<0.001)$.

\section{Laboratory parameters according to clinical severity on admission}

The laboratory parameters of the patients in each group are shown in Fig. 1. The coagulation profiles and levels of hematologic parameters, biochemical markers, inflammation markers, cardiac markers, and a molecular marker significantly differed according to clinical severity. Hb, Hct, LYM\% levels and PLT counts were lower in the moderate and severe groups than in the mild group $(P<0.001)$. WBC count, aPTT, PT INR, ALT, AST, Cr, K, Lac, CK-MB, and Tnl levels were significantly higher in the severe group than in the mild and moderate groups $(P<0.001)$. The Glu level increased with clinical severity $(P<0.001)$. In con- trast, albumin and $\mathrm{Na}$ levels were significantly lower in the moderate and severe groups than in the mild group $(P<0.001)$. T$\mathrm{BIL}, \mathrm{Cl}$, and $\mathrm{TnT}$ levels and $\mathrm{Ct}$ values of RdRP in LRT specimens were not associated with clinical severity.

The proportions of patients with abnormal laboratory parameter results were determined according to the reference values for the parameters (Table 2). The proportions of patients with abnormal LYM\%, albumin, AST, and CRP levels were significantly higher in the moderate and severe groups than in the mild group. There was no difference in the proportions of patients with abnormal Lac $(P=0.226)$, LD $(P=0.590)$, and TnT $(P=0.279)$ according to clinical severity.

\section{Correlation between laboratory parameters and clinical severity}

Multinomial regression analyses showed significant changes in the $\mathrm{OR}$ for each laboratory parameter with clinical severity in hospitalized COVID-19 patients (Table 3). For every unit increase in WBC, PT INR, PCT, CRP, and $\mathrm{Cr}$ levels, the risk of being in the moderate group was $1.189,2.384,1.417,1.225$, and 1.306 times higher, respectively, than the chance of being in the mild group. For every unit increase in WBC, PT INR, PCT, CRP, Cr, K, Lac, and Tnl, the risk of being in the severe group was 1.332, $3.127,1.462,1.227,1.433,1.589,1.519$, and 1.320 times higher, respectively, than the chance of being in the mild group. 
Table 2. Comparison of abnormal proportions of laboratory parameters based on clinical severity

\begin{tabular}{|c|c|c|c|c|c|}
\hline \multirow{2}{*}{ Variable } & \multirow{2}{*}{ Reference range } & \multicolumn{3}{|c|}{ Abnormal proportion according to clinical severity on admission, N / total N (\%) } & \multirow{2}{*}{$P$} \\
\hline & & Mild $(\mathrm{N}=1,612)$ & Moderate $(\mathrm{N}=294)$ & Severe $(N=46)$ & \\
\hline $\mathrm{Hb}(\mathrm{g} / \mathrm{dL})$ & $\begin{array}{l}\text { M 12.9-16.9 } \\
\text { F 11.5-15.5 }\end{array}$ & $427 / 1,608(26.6)$ & $125 / 294(42.5)$ & $20 / 43(46.5)$ & $<0.001$ \\
\hline Hct (\%) & $\begin{array}{l}\text { M 37.9-49.1 } \\
\text { F 32-43 }\end{array}$ & $344 / 1,609(21.4)$ & $114 / 293(38.9)$ & $24 / 43(55.8)$ & $<0.001$ \\
\hline LYM (\%) & $21-51$ & $410 / 1,606(25.5)$ & 208/291 (71.5) & $34 / 41(82.9)$ & $<0.001$ \\
\hline $\operatorname{PLT}\left(\times 10^{9} / \mathrm{L}\right)$ & $140-380$ & $232 / 1,609(14.4)$ & $85 / 294(28.9)$ & $16 / 42(38.1)$ & $<0.001$ \\
\hline $\mathrm{WBC}\left(\times 10^{9} / \mathrm{L}\right)$ & $3.6-9.6$ & $289 / 1,609(18.0)$ & $87 / 294(29.6)$ & $17 / 42(40.5)$ & $<0.001$ \\
\hline aPT (sec) & $28-45$ & $467 / 895(52.2)$ & $150 / 202(74.3)$ & 20/34 (58.8) & $<0.001$ \\
\hline PT INR & $0.8-1.2$ & $44 / 902(4.9)$ & $31 / 205(15.1)$ & $17 / 34(50.0)$ & $<0.001$ \\
\hline Albumin (g/dL) & $3.5-5.1$ & 146/1,596 (9.2) & $147 / 279(52.7)$ & $32 / 41(78.1)$ & $<0.001$ \\
\hline ALT (IU/L) & $<40$ & 212/1,607 (13.2) & 69/291 (23.7) & $17 / 41(41.5)$ & $<0.001$ \\
\hline AST (IU/L) & $<35$ & $313 / 1,608(19.5)$ & $176 / 292(60.3)$ & $33 / 41(80.5)$ & $<0.001$ \\
\hline $\mathrm{T}-\mathrm{BIL}$ (mg/dL) & $<1.2$ & 64/1,601 (4.0) & 40/277 (14.4) & $8 / 41(19.5)$ & $<0.001$ \\
\hline BUN (mg/dL) & $8.0-23.0$ & $243 / 1,608(15.1)$ & $105 / 294(35.7)$ & $20 / 42(47.6)$ & $<0.001$ \\
\hline $\mathrm{Cr}(\mathrm{mg} / \mathrm{dL})$ & $0.5-1.2$ & $160 / 1,605(10.0)$ & $76 / 293(25.9)$ & 20/42 (47.6) & $<0.001$ \\
\hline $\mathrm{CRP}$ (mg/dL) & $<0.5$ & $654 / 1,579(41.0)$ & $273 / 286(95.5)$ & $38 / 41$ (92.7) & $<0.001$ \\
\hline Glu (mg/dL) & $74-109$ & $728 / 1,506(48.3)$ & 196/271 (72.3) & $28 / 33(84.9)$ & $<0.001$ \\
\hline $\mathrm{Lac}(\mathrm{mmol} / \mathrm{L})$ & $0.7-2.1$ & $237 / 525(45.1)$ & $48 / 130(37.0)$ & 9/19 (47.4) & 0.226 \\
\hline LD (IU/L) & $<250$ & $1,376 / 1,458(94.4)$ & 216/232 (93.1) & $32 / 33(97.0)$ & 0.590 \\
\hline $\mathrm{Na}(\mathrm{mEq} / \mathrm{L})$ & $135-148$ & 103/1,601 (6.4) & $94 / 289(32.5)$ & $14 / 41$ (34.2) & $<0.001$ \\
\hline $\mathrm{Cl}(\mathrm{mEq} / \mathrm{L})$ & $98-110$ & 127/1,077 (11.8) & $68 / 229(29.7)$ & $8 / 33(24.2)$ & $<0.001$ \\
\hline$K(\mathrm{mEq} / \mathrm{L})$ & $3.5-5.1$ & $153 / 1,598(9.6)$ & 70/288 (24.3) & $15 / 41(36.6)$ & $<0.001$ \\
\hline PCT (mcg/L) & $<0.5$ & $17 / 639(2.7)$ & 23/158 (14.6) & $11 / 22(50.0)$ & $<0.001$ \\
\hline CK-MB ( $\mu \mathrm{g} / \mathrm{L})$ & $<3.6$ & 29/653 (4.4) & 33/184 (17.9) & $13 / 28(46.4)$ & $<0.001$ \\
\hline $\operatorname{Tnl}(\mu \mathrm{g} / \mathrm{L})$ & $<0.3$ & 6/641 (0.9) & $7 / 162(4.3)$ & 9/28 (32.1) & $<0.001$ \\
\hline $\operatorname{TnT}(\mu \mathrm{g} / \mathrm{L})$ & $<0.1$ & 2/18 (11.1) & $6 / 22(27.3)$ & $1 / 2(50.0)$ & 0.279 \\
\hline
\end{tabular}

Abbreviations: ALT, alanine aminotransferase; aPTT, activated partial thromboplastin time; AST, aspartate aminotransferase; BUN, blood urea nitrogen; CKMB, creatinine kinase MB isoenzyme; Cl, chloride; Cr, creatinine; CRP, C-reactive protein; F, female; Glu, glucose; K, potassium; Lac, lactate; LD, lactate dehydrogenase; LYM, Iymphocyte; M, male: Na, sodium; PCT, procalcitonin; PLT, platelet; PT INR, prothrombin time international normalized ratio; T-BIL, total bilirubin; Tnl, troponin I; TnT, troponin T; WBC, white blood cell.

In contrast, for every unit increase in the levels of $\mathrm{Hb}$, LYM\%, albumin, $\mathrm{K}$, and $\mathrm{Na}$, the risk of being in the moderate group was $0.855,0.880,0.084,0.656$, and 0.860 times lower, respectively, than the chance of being in the mild group. For every unit increase in the LYM\%, albumin, $\mathrm{Na}$, and Ct values of the RdRP gene in URT specimens, the risk of being in the severe group was $0.849,0.036,0.868$, and 0.883 times lower, respectively, than the chance of being in the mild group. Prolonged PT $(P<$ $0.001)$, hypoalbuminemia $(P<0.001)$, and abnormal $K$ levels $(P=0.003)$ were significantly correlated with severe disease.

We performed qualitative multinomial logistic regression analyses of the laboratory parameters (classified as normal vs. ab- normal according to the reference values) (Table 4). Abnormal laboratory parameters results showed significant correlations with disease severity. Abnormal levels of LYM\%, PT INR, albumin, AST, and PCT were associated with a 7.310, 3.474, 11.060, 6.277, and 6.234 times increased risk of moderate disease severity, respectively, as compared to the mild group. Abnormal levels of LYM\%, PT INR, albumin, AST, and PCT levels were associated with a $14.169,19.500,35.312,17.037$, and 36.588 times increased risk of severe disease, respectively, as compared to the mild group. Abnormal CRP values were more often observed in the moderate group than in the mild group (OR 30.280, 95\% Cl: 17.205-53.289, $P<0.001)$. Abnormal CK-MB and Tnl lev- 
Table 3. Multinomial logistic regression analysis of laboratory parameters for clinical severity

\begin{tabular}{|c|c|c|c|c|}
\hline \multirow{2}{*}{ Variable } & \multicolumn{2}{|c|}{ Mild vs. moderate } & \multicolumn{2}{|c|}{ Mild vs. severe } \\
\hline & OR (95\% Cl) & $P$ & OR (95\% Cl) & $P$ \\
\hline $\mathrm{Hb}(\mathrm{g} / \mathrm{dL})$ & $0.855(0.797-0.917)$ & $<0.001$ & $0.858(0.725-1.014)$ & 0.073 \\
\hline Hct (\%) & $0.934(0.911-0.957)$ & $<0.001$ & $0.943(0.889-1.000)$ & 0.049 \\
\hline LYM (\%) & $0.880(0.866-0.894)$ & $<0.001$ & $0.849(0.816-0.884)$ & $<0.001$ \\
\hline $\operatorname{PLT}\left(\times 10^{9} / \mathrm{L}\right)$ & $0.996(0.995-0.998)$ & $<0.001$ & $0.996(0.992-1.000)$ & 0.058 \\
\hline WBC $\left(\times 10^{9} / L\right)$ & $1.189(1.138-1.243)$ & $<0.001$ & $1.332(1.244-1.428)$ & $<0.001$ \\
\hline aPT (sec) & $1.100(1.071-1.13)$ & $<0.001$ & $1.141(1.104-1.18)$ & $<0.001$ \\
\hline PT INR & $2.384(1.543-3.684)$ & $<0.001$ & $3.127(1.897-5.156)$ & $<0.001$ \\
\hline Albumin (g/dL) & $0.084(0.062-0.113)$ & $<0.001$ & $0.036(0.02-0.064)$ & $<0.001$ \\
\hline ALT (IU/L) & $1.007(1.003-1.01)$ & $<0.001$ & $1.009(1.005-1.012)$ & $<0.001$ \\
\hline AST (IU/L) & $1.020(1.015-1.025)$ & $<0.001$ & $1.021(1.017-1.026)$ & $<0.001$ \\
\hline BUN (mg/dL) & $1.058(1.046-1.071)$ & $<0.001$ & $1.068(1.051-1.085)$ & $<0.001$ \\
\hline $\mathrm{Cl}(\mathrm{mEq} / \mathrm{L})$ & $0.960(0.93-0.992)$ & 0.014 & $1.016(0.935-1.104)$ & 0.713 \\
\hline $\mathrm{Cr}(\mathrm{mg} / \mathrm{dL})$ & $1.306(1.157-1.473)$ & $<0.001$ & $1.433(1.230-1.670)$ & $<0.001$ \\
\hline CRP (mg/dL) & $1.225(1.195-1.255)$ & $<0.001$ & $1.227(1.191-1.263)$ & $<0.001$ \\
\hline Glu (mg/dL) & $1.007(1.006-1.009)$ & $<0.001$ & $1.010(1.007-1.013)$ & $<0.001$ \\
\hline$K(m E q / L)$ & $0.656(0.517-0.833)$ & 0.001 & 1.589 (1.173-2.153) & 0.003 \\
\hline Lac (mmol/L) & $1.096(0.935-1.284)$ & 0.258 & 1.519 (1.253-1.842) & $<0.001$ \\
\hline LD (IU/L) & $1.000(1.000-1.000)$ & 0.006 & $1.000(1.000-1.000)$ & 0.006 \\
\hline $\mathrm{Na}(\mathrm{mEg} / \mathrm{L})$ & $0.860(0.833-0.887)$ & $<0.001$ & $0.868(0.811-0.929)$ & $<0.001$ \\
\hline PCT (mcg/L) & $1.417(1.115-1.802)$ & 0.004 & 1.462 (1.119-1.909) & 0.005 \\
\hline $\mathrm{T}-\mathrm{BIL}(\mathrm{mg} / \mathrm{dL})$ & $1.058(0.97-1.153)$ & 0.204 & $1.076(0.967-1.197)$ & 0.177 \\
\hline CK-MB ( $\mu \mathrm{g} / \mathrm{L})$ & $1.048(1.006-1.092)$ & 0.023 & $1.065(1.018-1.113)$ & 0.006 \\
\hline $\operatorname{Tnl}(\mu \mathrm{g} / \mathrm{L})$ & $1.163(0.929-1.455)$ & 0.188 & $1.320(1.043-1.669)$ & 0.021 \\
\hline $\operatorname{TnT}(\mu \mathrm{g} / \mathrm{L})$ & $1.298(0.446-3.777)$ & 0.633 & $1.358(0.204-9.028)$ & 0.752 \\
\hline RdRP, Ct (URT) & $0.955(0.925-0.986)$ & 0.005 & $0.883(0.83-0.939)$ & $<0.001$ \\
\hline RdRP, Ct (LRT) & $0.951(0.892-1.013)$ & 0.120 & $0.936(0.822-1.065)$ & 0.315 \\
\hline
\end{tabular}

Abbreviations: ALT, alanine aminotransferase; aPTT, activated partial thromboplastin time; AST, aspartate aminotransferase; BUN, blood urea nitrogen; CI, confidence interval; CK-MB, creatinine kinase MB isoenzyme; Cl, chloride; Cr, creatinine; CRP, C-reactive protein; Ct, cyclic threshold; Glu, glucose; K, potassium; Lac, lactate; LD, lactate dehydrogenase; LRT, lower respiratory tract; LYM, lymphocyte; Na, sodium; OR, odds ratio; PCT, procalcitonin; PLT, platelet; PT INR, prothrombin time international normalized ratio; RdRP, RNA-dependent RNA polymerase gene; T-BIL, total bilirubin; Tnl, troponin I; TnT, troponin T; URT, upper respiratory tract; WBC, white blood cell.

els indicated severe disease more significantly than mild disease (OR, 18.648, 95\% Cl: 8.125-42.8, $P<0.001$ and OR, 44.561, 95\% Cl: $14.072-141.11, P<0.001$, respectively).

\section{Hypoalbuminemia and lymphopenia are associated with severe COVID-19}

The frequencies of hypoalbuminemia and relative lymphopenia are shown in Table 5. In the severe group, 39 (95\%) patients had either hypoalbuminemia or relative lymphopenia. The ORs of combined lymphopenia and hypoalbuminemia were 15.228
(95\% Cl: 10.988-21.104, $P<0.001)$ and $36.474(95 \% \mathrm{Cl}$ : 18.413-72.251, $P<0.001)$ in the moderate and severe groups, respectively. In the ROC curves, the area under the curve (AUC) for albumin was 0.877 (95\% Cl: 0.831-0.922, $P<0.001)$, with a sensitivity of $78.0 \%$ and a specificity of $84.8 \%$, and that for LYM\% was 0.824 (95\% Cl: 0.752-0.896, $P<0.001$ ), with a sensitivity of $73.2 \%$ and a specificity of $85.8 \%$. The optimal cutoff levels of albumin and LYM\% to predict severe COVID-19 were $3.44 \mathrm{~g} / \mathrm{dL}$ and $14.8 \%$, respectively. 
Table 4. Multinomial logistic regression analysis of abnormal laboratory parameters for clinical severity (abnormal vs. normal)

\begin{tabular}{|c|c|c|c|c|}
\hline \multirow{2}{*}{ Variable } & \multicolumn{2}{|c|}{ Mild vs. moderate } & \multicolumn{2}{|c|}{ Mild vs. severe } \\
\hline & OR (95\% Cl) & $P$ & OR (95\% Cl) & $P$ \\
\hline $\mathrm{Hb}(\mathrm{g} / \mathrm{dL})$ & 2.046 (1.583-2.643) & $<0.001$ & 2.405 (1.308-4.424) & 0.005 \\
\hline Hct (\%) & $2.342(1.800-3.048)$ & $<0.001$ & $4.645(2.515-8.579)$ & $<0.001$ \\
\hline LYM (\%) & $7.310(5.536-9.654)$ & $<0.001$ & $14.169(6.233-32.208)$ & $<0.001$ \\
\hline $\operatorname{PLT}\left(\times 10^{9} / \mathrm{L}\right)$ & 2.414 (1.810-3.219) & $<0.001$ & 3.653 (1.930-6.914) & $<0.001$ \\
\hline $\mathrm{WBC}\left(\times 10^{9} / \mathrm{L}\right)$ & $1.920(1.450-2.542)$ & $<0.001$ & $3.106(1.656-5.827)$ & $<0.001$ \\
\hline aPT (sec) & $0.378(0.269-0.532)$ & $<0.001$ & $0.764(0.381-1.531)$ & 0.448 \\
\hline PT INR & $3.474(2.133-5.657)$ & $<0.001$ & $19.500(9.328-40.764)$ & $<0.001$ \\
\hline Albumin (g/dL) & $11.060(8.275-14.783)$ & $<0.001$ & 35.312 (16.533-75.419) & $<0.001$ \\
\hline ALT (IU/L) & 2.045 (1.506-2.778) & $<0.001$ & $4.661(2.463-8.821)$ & $<0.001$ \\
\hline AST (IU/L) & 6.277 (4.816-8.181) & $<0.001$ & $17.067(7.806-37.312)$ & $<0.001$ \\
\hline BUN (mg/dL) & 3.121 (2.371-4.108) & $<0.001$ & $5.107(2.745-9.500)$ & $<0.001$ \\
\hline $\mathrm{Cl}(\mathrm{mEq} / \mathrm{L})$ & 3.159 (2.252-4.433) & $<0.001$ & $2.394(1.057-5.421)$ & 0.036 \\
\hline $\mathrm{Cr}(\mathrm{mg} / \mathrm{dL})$ & $3.163(2.324-4.304)$ & $<0.001$ & $8.210(4.385-15.372)$ & $<0.001$ \\
\hline $\mathrm{CRP}(\mathrm{mg} / \mathrm{dL})$ & $30.280(17.205-53.289)$ & $<0.001$ & $18.264(5.614-59.416)$ & $<0.001$ \\
\hline Glu (mg/dL) & $2.793(2.101-3.713)$ & $<0.001$ & 5.985 (2.299-15.582) & $<0.001$ \\
\hline $\mathrm{K}(\mathrm{mEq} / \mathrm{L})$ & $3.033(2.210-4.162)$ & $<0.001$ & $5.449(2.825-10.510)$ & $<0.001$ \\
\hline $\mathrm{Lac}(\mathrm{mmol} / \mathrm{L})$ & $0.711(0.479-1.056)$ & 0.091 & $1.094(0.437-2.736)$ & 0.848 \\
\hline LD (IU/L) & $0.805(0.462-1.401)$ & 0.442 & $1.907(0.257-14.130)$ & 0.528 \\
\hline $\mathrm{Na}(\mathrm{mEq} / \mathrm{L})$ & 7.011 (5.107-9.625) & $<0.001$ & 7.541 (3.837-14.821) & $<0.001$ \\
\hline PCT (mcg/L) & 6.234 (3.241-11.988) & $<0.001$ & 36.588 (13.944-96.005) & $<0.001$ \\
\hline T-BIL (mg/dL) & $4.053(2.668-6.157)$ & $<0.001$ & $5.822(2.585-13.112)$ & $<0.001$ \\
\hline CK-MB ( $\mu \mathrm{g} / \mathrm{L})$ & 4.702 (2.769-7.986) & $<0.001$ & $18.648(8.125-42.800)$ & $<0.001$ \\
\hline $\operatorname{Tnl}(\mu \mathrm{g} / \mathrm{L})$ & 4.780 (1.584-14.423) & 0.006 & 44.561 (14.072-141.110) & $<0.001$ \\
\hline $\operatorname{TnT}(\mu \mathrm{g} / \mathrm{L})$ & $3.000(0.525-17.159)$ & 0.217 & $8.000(0.347-184.364)$ & 0.194 \\
\hline RdRP, Ct (URT) & 1.791 (1.238-2.592) & 0.002 & 2.338 (0.919-5.952) & 0.075 \\
\hline RdRP, Ct (LRT) & 3.161 (1.737-5.754) & $<0.001$ & 3.035 (0.792-11.634) & 0.105 \\
\hline
\end{tabular}

Abbreviations: ALT, alanine aminotransferase; aPTT, activated partial thromboplastin time; AST, aspartate aminotransferase; BUN, blood urea nitrogen; $\mathrm{Cl}$, confidence interval; CK-MB, creatinine kinase MB isoenzyme; $\mathrm{Cl}$, chloride; $\mathrm{Cr}$, creatinine; CRP, C-reactive protein; Ct, cyclic threshold; Glu, glucose; K, potassium; Lac, lactate; LD, lactate dehydrogenase; LRT, lower respiratory tract; LYM, lymphocyte; Na, sodium; OR, odds ratio; PCT, procalcitonin; PLT, platelet; PT INR, prothrombin time international normalized ratio; RdRP, RNA-dependent RNA polymerase gene; T-BIL, total bilirubin; Tnl, troponin I; TnT, troponin T; URT, upper respiratory tract; WBC, white blood cell.

Table 5. Hypoalbuminemia and relative lymphopenia as laboratory parameters associated with severe COVID-19

\begin{tabular}{|c|c|c|c|c|c|c|c|}
\hline \multirow{2}{*}{ Variable } & \multicolumn{3}{|c|}{ N/total N (\%) } & \multicolumn{2}{|c|}{ Mild vs. moderate } & \multicolumn{2}{|l|}{ Mild vs. severe } \\
\hline & Mild & Moderate & Severe & OR (95\% Cl) & $P$ & OR $(95 \% \mathrm{Cl})$ & $P$ \\
\hline $\begin{array}{l}\text { Hypoalbuminemia } \\
(<3.5 \mathrm{~g} / \mathrm{dL})\end{array}$ & $141 / 1,596(8.8)$ & $147 / 279(52.7)$ & $32 / 41(78.1)$ & $10.433(7.833-13.895)$ & $<0.001$ & $23.846(14.432-45.741)$ & $<0.001$ \\
\hline Lymphopenia (<21\%) & $343 / 1,606(21.5)$ & 206/291 (70.8) & $34 / 41(82.9)$ & 8.924 (6.748-11.801) & $<0.001$ & 17.885 (7.860-40.697) & $<0.001$ \\
\hline $\begin{array}{l}\text { Hypoalbuminemia+ } \\
\text { lymphopenia }\end{array}$ & $80 / 1,591(5.0)$ & $124 / 276(44.6)$ & $27 / 41(65.9)$ & $15.228(10.988-21.104)$ & $<0.001$ & $36.474(18.413-72.251)$ & $<0.001$ \\
\hline
\end{tabular}

Abbreviations: $\mathrm{Cl}$, confidence interval; OR, odds ratio. 


\section{DISCUSSION}

We found old age, male sex, relative lymphopenia, PT prolongation, and hypoalbuminemia to be significant indicators of COVID-19 severity. Patients with both hypoalbuminemia and lymphopenia showed an increased risk of severe COVID-19.

With respect to general characteristics, old age and male sex were significantly associated with severe COVID-19, in line with previous results $[3,4,13]$. Old age is often associated with compromised immune function and the presence of underlying comorbidities, which may lead to poor patient outcomes [14]. Recently, Takahashi, et al. [15] showed key differences in T-cell responses and cytokine levels during the course of COVID-19 in male and female patients. These differences may explain the higher vulnerability of men to COVID-19.

Comparison of the hematologic parameters revealed significant decreases in $\mathrm{Hb}$, Hct, LYM\%, and PLT count in the moderate and severe groups as compared to the mild group. Patients with severe disease on admission showed a higher WBC count; this is consistent with the results of a Chinese study [16]. In particular, the ORs of LYM\% differed significantly between the moderate and severe groups and the mild group in both quantitative and qualitative analyses. A previous study reported a clear correlation between lymphopenia and disease severity [17]. In a meta-analysis of 13 cases, Zhao, et al. [18] found that lymphopenia, defined as a lymphocyte count of $<1.5 \times 10^{9} / \mathrm{L}$, was associated with a three-fold increase in severe SARS-CoV-2 infection risk and that lymphocyte count and lymphopenia may serve as biomarkers for the rapid identification of COVID-19 patients with a more severe clinical presentation. Tan, et al. [19] reported that LYM\% can be used as a reliable indicator for classifying COVID-19 patients into moderate, severe, and critical disease groups. Pronounced lymphocyte depletion and persistent low lymphocyte counts may be related to critical illness and unfavorable outcomes [8-10]. Similar to SARS-CoV, SARS-CoV-2 may act mainly on lymphocytes, especially, T lymphocytes [4]. The mechanisms of action include direct infection and lymphocyte destruction, cytokine-mediated lymphocyte destruction, and the inhibition of lymphocytes by metabolites, such as Lac [19-22]. Virus particles spread through the respiratory mucosa and infect other cells, induce a cytokine storm in the body, generate a series of immune responses, and cause changes in peripheral WBCs and immune cells, such as lymphocytes.

Abnormal coagulation parameters were more frequently observed in the moderate and severe groups than in the mild group. Prominent changes in the coagulation profile, including those in
aPTT and PT INR, were noted in the three study groups. Liao, et al. [23] reported that the severity of coagulopathy was associated with the severity of COVID-19 in 380 patients. Tang, et al. [24] found that increased D-dimer and fibrin degradation product levels as well as prolonged PT on admission are related to poor prognosis. In a meta-analysis of 1,105 patients, Xiong, et al. [25] reported that PT and D-dimer levels were significantly higher in patients with severe COVID-19 than in those with mild disease.

Dysfunctional coagulation is considered an important risk factor for severe disease and death [26]. Coagulation activation may be related to the sustained inflammatory response. In severe infection, coagulation activation leads to an increased risk of the excessive consumption of coagulation factors with attendant disseminated intravascular coagulation, resulting in an increased fatality rate [27]. Considerable evidence has shown that COVID-19 patients show abnormal coagulation at both clinical presentation and laboratory examination [24, 26]. COVID-19-associated lung tissue damage may induce the release of tissue factors in the circulation and promote secondary fibrinolysis via the exogenous coagulation pathway [8]. Although the specific underlying mechanisms remain unclear, COVID-19 obviously involves potentially deleterious hemostasis/coagulation and inflammation processes.

COVID-19 is a systemic disease that affects several organs, including the liver, heart, and kidneys [20, 28-30]. The incidence of liver function abnormalities in COVID-19 patients is reported to be $14 \%-53 \%$ [31]. In our study, hypoalbuminemia and abnormal AST levels were prominent in severe COVID-19 patients. Albumin levels were inversely correlated with COVID-19 severity, and $78.1 \%$ of the patients in the severe group showed hypoalbuminemia. Abnormal albumin level was the most frequent abnormal liver biochemical indicator on admission, and approximately $20 \%$ of all patients with acute medical admissions had reduced albumin levels [32, 33]. Decreased albumin level generally indicates depressed hepatic synthesis, poor nutritional status, or negative acute-phase reactants due to downregulation by inflammatory cytokines [34]. Studies in various clinical settings and major disease categories have shown that hypoalbuminemia is associated with increased morbidity and mortality $[35,36]$. Therefore, the albumin level may be used as a clinical marker to identify patients at high risk of developing severe COVID-19. Although there is currently no evidence that prevention or treatment of hypoalbuminemia improves the prognosis of COVID-19 patients, a decline in the albumin level may be related to the severity of COVID-19. 
The proportion of patients with both hypoalbuminemia and relative lymphopenia was substantially higher in the severe group than in the mild group (65.9\% vs. 5.0\%). Although further analysis for disease progression is needed, most patients with an albumin level above $3.44 \mathrm{~g} / \mathrm{dL}$ and LYM\% above 14.8\% on admission may not need intensive treatment. Combined hypoalbuminemia and lymphopenia may represent systemic inflammation and be associated with an increased risk of severe disease.

Although numerous studies have reported on laboratory indicators of COVID-19, most of them were meta-analyses using laboratory parameters of Chinese patients [8, 9, 32]. Meta-analysis results may be limited by the selection of an incomplete set of studies; studies lacking internal, external, construct, and statistical conclusion validity; studies with relatively small sample sizes; and heterogeneity of methods used in included studies, all of which may lead to erroneous inferences [37]. Our study included a relatively large number of patients, and the heterogeneity of the enrolled patients was relatively low.

The present study had some limitations. First, asymptomatic or minimally symptomatic patients with no concurrent disease were treated in community treatment centers and were excluded; only hospitalized patients were included. Thus, the results may be biased, with a tendency to reveal more serious consequences in terms of clinical and laboratory parameters. Second, not all tests were performed in all patients. There were differences in the laboratory tests according to the medical staff or clinical severity. Third, the $95 \% \mathrm{Cls}$ for OR estimates were rather wide in the multinomial regression analysis using qualitative variables, including PT INR, albumin, and LYM\%. This may have been due to the wide range of values for these variables and the small sample size of the severe group. Further analysis using a large dataset may be helpful. Fourth, the reagents and instruments used in the laboratories of the nine hospitals were different. However, most laboratories in Korea participate in the Korean Laboratory Accreditation Program by the Laboratory Medicine Foundation and the Korean External Quality Assessment Scheme for laboratory standardization. Thus, the differences in procedures among these laboratories are not considered significant [38].

The present study focused on laboratory parameters that could predict clinical COVID-19 severity to classify and properly treat hospitalized COVID-19 patients. Various laboratory parameters, including hematologic, coagulation, biochemical, inflammatory, and cardiac markers, can be used to predict the severity of COVID-19. Although abnormalities in these parameters are not specific to COVID-19, they can help predict clinical severity in COVID-19 patients on admission. Laboratory tests are simple and inexpensive tools to provide objective information about the severity in COVID-19 patients. thus, these test results should be closely monitored and used to take timely measures.

\section{ACKNOWLEDGEMENTS}

None.

\section{AUTHOR CONTRIBUTIONS}

Yoo E and Chang SH designed the study, analyzed the data, and wrote the manuscript; Song D, Lee CH, Cheong GY, Park S, Lee $\mathrm{JH}$, and Lee S collected and analyzed the data; Kwak SG performed the statistical analysis; Jeon $\mathrm{C}$ and Song $\mathrm{K}$ supervised the study design and reviewed and commented on the manuscript; all authors reviewed and approved the manuscript.

\section{CONFLICTS OF INTEREST}

No potential conflicts of interest relevant to this article are reported.

\section{RESEARCH FUNDING}

This work was supported by the Research Program of Medicity Daegu Council funded by Daegu Metropolitan City (fund code COVID19_DM06).

\section{ORCID}

Eun-Hyung Yoo

Soon Hee Chang

Do-Young Song

Chae Hoon Lee

Gyu Young Cheong

Sunggyun Park

Jae Hee Lee

Sooin Lee

Sang-Gyu Kwak

Chang-Ho Jeon

Kyung Eun Song https://orcid.org/0000-0002-6854-8932 https://orcid.org/0000-0003-3031-2200 https://orcid.org/0000-0002-5474-8327 https://orcid.org/0000-0001-7722-9004 https://orcid.org/0000-0003-1852-1245 https://orcid.org/0000-0002-7094-2808 https://orcid.org/0000-0002-0402-4718 https://orcid.org/0000-0001-9326-2752 https://orcid.org/0000-0003-0398-5514 https://orcid.org/0000-0002-7450-7117 https://orcid.org/0000-0003-1628-4741

\section{REFERENCES}

1. Xu Z, Shi L, Wang Y, Zhang J, Huang L, Zhang C, et al. Pathological findings of COVID-19 associated with acute respiratory distress syn- 
drome. Lancet Respir Med 2020;8:420-2.

2. Kim IH, Kang BH, Seo SH, Park YE, Kim GJ, Lee SW, et al. Early Laboratory Preparedness of the Korea Disease Control and Prevention Agency and Response to Unknown Pneumonia Outbreak from Wuhan, China, in January 2020. Ann Lab Med 2021;41:532-9.

3. Argenziano MG, Bruce SL, Slater CL, Tiao JR, Baldwin MR, Barr RG, et al. Characterization and clinical course of 1000 patients with coronavirus disease 2019 in New York: retrospective case series. BMJ 2020; 369:m1996.

4. Chen N, Zhou M, Dong X, Qu J, Gong F, Han Y, et al. Epidemiological and clinical characteristics of 99 cases of 2019 novel coronavirus pneumonia in Wuhan, China: a descriptive study. Lancet 2020;395:507-13.

5. Sung HK, Kim JY, Heo J, Seo H, Jang YS, Kim H, et al. Clinical course and outcomes of 3,060 patients with coronavirus disease 2019 in Korea, January-May 2020. J Korean Med Sci 2020;35:e280.

6. Central Disaster Management Headquarters. Patient Treatment and Management, http://ncov.mohw.go.kr/en/baroView.do?brdld=11\&brdGu bun=112\&dataGubun=\&ncvContSeq=\&contSeq=\&board_id=\&gubun= (Updated on June, 2020).

7. Kim JH, An JAR, Min PK, Bitton A, Gawande AA. How South Korea responded to the Covid-19 outbreak in Daegu. NEJM Catal Innov Care Deliv 2020;1:10.1056/CAT.20.0159.

8. Skevaki C, Fragkou PC, Cheng C, Xie M, Renz H. Laboratory characteristics of patients infected with the novel SARS-CoV-2 virus. J Infect 2020; 81:205-12.

9. Henry BM, de Oliveira MHS, Benoit S, Plebani M, Lippi G. Hematologic, biochemical and immune biomarker abnormalities associated with severe illness and mortality in coronavirus disease 2019 (COVID-19): a meta-analysis. Clin Chem Lab Med 2020;58:1021-8.

10. Fan BE, Chong VCL, Chan SSW, Lim GH, Lim KGE, Tan GB, et al. Hematologic parameters in patients with COVID-19 infection. Am J Hematol 2020;95:E131-4.

11. WHO Working Group on the Clinical Characterisation and Management of COVID-19 infection. A minimal common outcome measure set for COVID-19 clinical research. Lancet Infect Dis 2020;20:e192-7.

12. Scheffé $H$. A method for judging all contrasts in the analysis of variance. Biometrika 1953;40:87-104.

13. Yang J, Zheng Y, Gou X, Pu K, Chen Z, Guo Q, et al. Prevalence of comorbidities and its effects in patients infected with SARS-CoV-2: a systematic review and meta-analysis. Int J Infect Dis 2020;94:91-5.

14. Opal SM, Girard TD, Ely EW. The immunopathogenesis of sepsis in elderly patients. Clin Infect Dis 2005;41(S7):S504-12.

15. Takahashi T, Ellingson MK, Wong P, Israelow B, Lucas C, Klein J, et al. Sex differences in immune responses that underlie COVID-19 disease outcomes. Nature 2020;588:315-20.

16. Zhu B, Feng X, Jiang C, Mi S, Yang L, Zhao Z, et al. Correlation between white blood cell count at admission and mortality in COVID-19 patients: a retrospective study. BMC Infect Dis 2021;21:574.

17. Henry BM. COVID-19, ECMO, and lymphopenia: a word of caution. Lancet Respir Med 2020;8:e24.

18. Zhao Q, Meng M, Kumar R, Wu Y, Huang J, Deng Y, et al. Lymphopenia is associated with severe coronavirus disease 2019 (COVID-19) infections: A systemic review and meta-analysis. Int J Infect Dis 2020;96: 131-5.

19. Tan L, Wang Q, Zhang D, Ding J, Huang Q, Tang YQ, et al. Lymphopenia predicts disease severity of COVID-19: a descriptive and predictive study. Signal Transduct Target Ther 2020;5:33.

20. Zheng HY, Zhang M, Yang CX, Zhang N, Wang XC, Yang XP, et al. Ele- vated exhaustion levels and reduced functional diversity of $T$ cells in peripheral blood may predict severe progression in COVID-19 patients. Cell Mol Immunol 2020;17:541-3.

21. Zheng M, Gao Y, Wang G, Song G, Liu S, Sun D, et al. Functional exhaustion of antiviral lymphocytes in COVID-19 patients. Cell Mol Immunol 2020; 17:533-5.

22. Fischer K, Hoffmann P, VoelkI S, Meidenbauer N, Ammer J, Edinger M, et al. Inhibitory effect of tumor cell-derived lactic acid on human T cells. Blood 2007;109:3812-9.

23. Liao D, Zhou F, Luo L, Xu M, Wang H, Xia J, et al. Haematological characteristics and risk factors in the classification and prognosis evaluation of COVID-19: a retrospective cohort study. Lancet Haematol 2020;7: e671-8.

24. Tang N, Li D, Wang X, Sun Z. Abnormal coagulation parameters are associated with poor prognosis in patients with novel coronavirus pneumonia. J Thromb Haemost 2020;18:844-7.

25. Xiong M, Liang X, Wei YD. Changes in blood coagulation in patients with severe coronavirus disease 2019 (COVID-19): a meta-analysis. Br J Haematol 2020;189:1050-2.

26. Giannis D, Ziogas IA, Gianni P. Coagulation disorders in coronavirus infected patients: COVID-19, SARS-CoV-1, MERS-CoV and lessons from the past. J Clin Virol 2020;127:104362.

27. Han H, Yang L, Liu R, Liu F, Wu KL, Li J, et al. Prominent changes in blood coagulation of patients with SARS-CoV-2 infection. Clin Chem Lab Med 2020;58:1116-20.

28. Guan WJ, Ni ZY, Hu Y, Liang WH, Ou CQ, He JX, et al. Clinical characteristics of coronavirus disease 2019 in China. N Engl J Med 2020;382: 1708-20.

29. Huang C, Wang Y, Li X, Ren L, Zhao J, Hu Y, et al. Clinical features of patients infected with 2019 novel coronavirus in Wuhan, China. Lancet 2020;395:497-506.

30. Bivona G, Agnello L, Ciaccio M. Biomarkers for prognosis and treatment response in COVID-19 patients. Ann Lab Med 2021;41:540-8.

31. Zhang C, Shi L, Wang FS. Liver injury in COVID-19: management and challenges. Lancet Gastroenterol Hepatol 2020;5:428-30.

32. Wu Y, Li H, Guo X, Yoshida EM, Mendez-Sanchez N, Levi Sandri GB, et al. Incidence, risk factors, and prognosis of abnormal liver biochemical tests in COVID-19 patients: a systematic review and meta-analysis. Hepatol Int 2020;14:621-37.

33. Herrmann FR, Safran C, Levkoff SE, Minaker KL. Serum albumin level on admission as a predictor of death, length of stay, and readmission. Arch Intern Med 1992;152:125-30.

34. Lyons O, Whelan B, Bennett K, O'Riordan D, Silke B. Serum albumin as an outcome predictor in hospital emergency medical admissions. Eur J Intern Med 2010;21:17-20.

35. Li N, Qiao H, Guo JF, Yang HY, Li XY, Li SL, et al. Preoperative hypoalbuminemia was associated with acute kidney injury in high-risk patients following non-cardiac surgery: a retrospective cohort study. BMC Anesthesiol 2019;19:171.

36. Ablett AD, McCarthy K, Carter B, Pearce L, Stechman M, Moug S, et al. A practical risk scale for predicting morbidity and mortality in the emergency general surgical setting: A prospective multi-center study. Int J Surg 2018;60:236-44.

37. Lee YH. An overview of meta-analysis for clinicians. Korean J Intern Med 2018;33:277-83.

38. Jang MA, Yoon YA, Song J, Kim JH, Min WK, Lee JS, et al. Effect of accreditation on accuracy of diagnostic tests in medical laboratories. Ann Lab Med 2017;37:213-22. 